\title{
Partnerships between patients, medicine, and the biotechnology industry
}

\author{
Alastair Kent and Erik Tambuyzer
}

Tremendous advances have been made with respect to the scientific understanding of disease. In the developed world at least, many previously fatal or debilitating conditions are now treatable, but even so, there are still thousands of different conditions, all of which result in chronic ill health, disability, or premature death for which medicine offers little more than sympathy, social support, and in some cases, palliation of symptoms.

Some of the more common diseases, like cancer and Alzheimer's, affect many millions in their own right. Others are called rare, but are still responsible for an unimaginable weight of suffering and stress-not only for the affected patients as individuals, but also for those who may themselves be at risk but are as yet presymptomatic, for family members, and for others who provide care and support.

When such a condition has a childhood onset, the first difficulty has often been, for patients and parents, to get a diagnosis. Once a diagnosis has been made, then comes the search for information. For those with rare diseases, many of which have strange and unpronounceable names associated with the scientists and clinicians who first described them, the search for information can be problematic and frustrating. A patient can look to the family doctor for advice and support, but he or she may never have even heard of the condition in question. It is therefore not uncommon for doctors to come to rely on those with rare disorders for useful information about the illness rather than the other way around. These patients have a direct and immediate need to know about the latest research and treatment options.

As a result, throughout the developed world, patient groups have emerged to provide information, advice, and support for those affected. They have often gone on to develop specialist services not available

Alastair Kent is with the European Alliance of Genetic Support Groups and the Genetic Interest Group, UK (mail@org.uk).Erik Tambuyzer is vice president, corporate affairs Europe, Genzyme Corporation (Leuven, Belgium), and is a board member of EuropaBio, the European BioIndustries Association(etambuyzer@genzyme.com)

Table 1. Examples of research collaborations among patient groups/industry/academia.

Pharming (The Netherlands) and Genzyme (US), VSN and AMDA patient groups, and the University of Rotterdam are collaborating on a treatment for Pompe's disease, a lysosomal storage disorder.

Transgène (France) with the "Association Française contre les Myopathies" (AFM) are working on gene therapy for neuromuscular diseases.

Rhône-Poulenc Rorer and Cephalon are collaborating with the Universities of Amsterdam and Utrecht and the Dutch patient association for muscular disorders on motor neuron diseases.

Researchers in academia and clinical centers are funded by charities organized by patient groups such as Genethon in France and Telethon in Italy and more will surely follow.

The US National Institutes of Health are organizing rare disease research status workshops to link academia with industrial partners in biotechnology.

from statutory health services to fund basic and applied research into "their" condition (and in the case of the largest patient organizations, to undertake that research in institutes that they have developed and manage themselves). More recently, patient groups have become increasingly active in issues of national or supernational policymaking, sometimes acting on their own, but more often through the creation of patient alliances and umbrella organizations. In Europe, two of the best known of these are EAGS, the European Alliance of Genetic Support Groups (London) and Eurordis, the European Rare Disorders Organization (Paris). In other parts of the world, similar groups exist; US equivalents are the Alliance of Genetic Support Groups (Washington, D.C.) and NORD, the National Organization for Rare Disorders (New Fairfield, CT).

Recent European initiatives in which patient groups have played a significant role in public debate include the European Community biotechnology patent directive, the genetics research referendum in Switzerland, the discussion about transgenic animals in The Netherlands, the clinical trials directive, and the proposed regulation on orphan medicinal products, the outcome of which is awaited by many millions of European citizens for whom medicine currently has little to offer.

The driver for all these activities has been patient need, often associated with fund-raising to stimulate basic research long before there was any product or treatment possibility.
Patient groups, clinicians, and industry working together have also been crucial to the successful development of treatments for rare diseases. In the US, patient pressure resulted in 1983 in the US Orphan Drug Act; in March 1999, the European Parliament voted for an equivalent European regulation. The US Orphan Drug Act has resulted in over 180 medicines for rare conditions.

New partnerships are emerging between all the stakeholders in the process for research into treatment for many currently incurable or intractable conditions. These partnerships also include a new educational relationship once a new treatment for a rare condition is brought to market. Physicians, patient groups, and industry collaborate to identify patients, establ;ish efficientdiagnostics methods, as well as to perform monitoring and build databases.

In Europe, a number of interesting and fruitful partnerships among biotechnology companies, patient groups, and academia have sprung up despite the absence of relevant European orphan drug regulations. Some examples of these collaborations are listed in Table 1. We believe that the proposed European orphan drug regulation will greatly increase the number and quality of these partnerships in the future. Such partnerships do not arise spontaneously. They take time and energy and a great deal of money to develop. But for those living in the shadow of serious and chronic ill health with the consequent loss of quality of life that such illness brings, patient advocacy and collaborative efforts with industry provide yet another bit of light at the end of a very dark tunnel. /// 